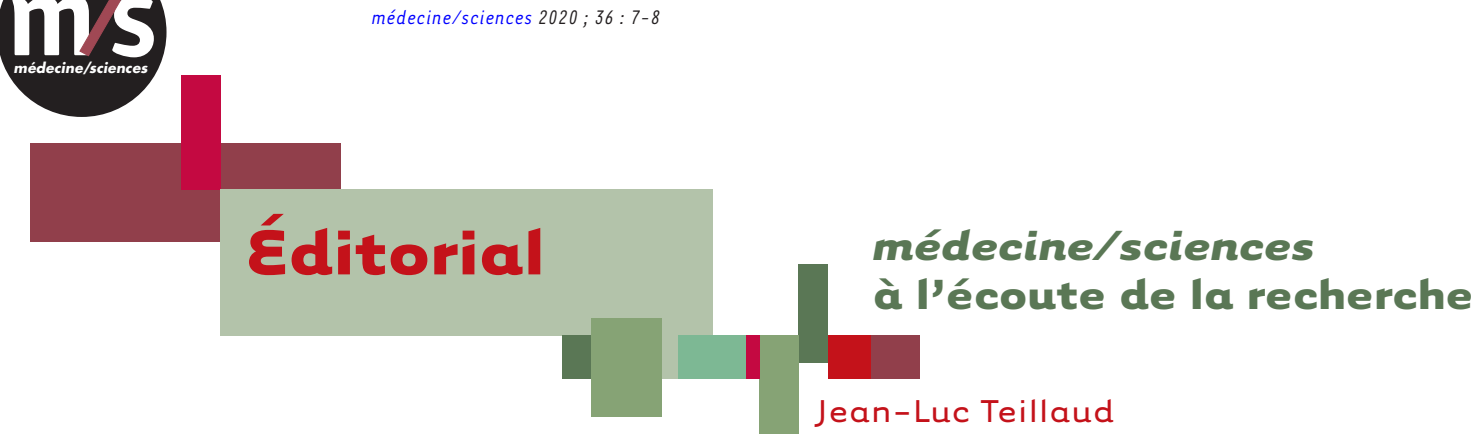

> Être à l'écoute de la recherche, pour médecine/sciences, c'est aimer la recherche, aimer naviguer aux frontières de la connaissance, accompagner nos collègues dans leurs découvertes, leurs questionnements, leurs hésitations et tâtonnements. Aimer la recherche, c'est également aimer la rigueur expérimentale, l'exigence et l'intégrité scientifique, qui se doivent d'accompagner la créativité et l'imagination; c'est aussi aimer la vivacité, voire la rugosité, de débats qui visent encore et encore à remettre en question des certitudes et des dogmes et à bouleverser des paradigmes dont nous savons tous qu'ils n'ont souvent qu'un temps, leur temps. Quel chercheur ne rêve-t-il pas de voir ses idées aboutir expérimentalement, ses résultats reproduits et commentés par ses pairs, ses résultats et leurs discours diffusés et expliqués? Quel clinicien ne pense-t-il pas qu'au travers de ses observations au lit du malade, il pourra se retourner vers un questionnement plus fondamental dont les réponses viendront enrichir en retour ses possibilités d'intervention thérapeutique?

La recherche est porteuse de valeurs essentielles: I'acceptation du regard croisé de l'autre, qui valide ou invalide et discute théories et données expérimentales; un internationalisme des échanges de la pensée, qui abat par essence les murs que certains pourraient vouloir de nouveau ériger, internationalisme que nous tous connaissons bien et qui nous donne un regard aigu sur l'évolution du monde dans sa globalité, une connaissance précise des puissances scientifiques émergentes; la recherche biomédicale, c'est également la capacité de relier, de mettre en réseau, des chercheurs venus de champs scientifiques différents - mathématiques, sciences humaines et sociales, physique et chimie... - pour engendrer la force créatrice apportée par la multidisciplinarité, dont la vigueur hybride est issue de la rencontre parfois aléatoire et non décrétée de femmes et d'hommes venus de domaines longtemps parallèles; mais la recherche est également une école, école de la rigueur, où les faux-semblants sont rapidement rejetés, école où il faut... aimer la découverte, cette excitation frémissante devant un nouveau résultat, devant une idée qui germe, école du risque et de l'obstination butée au risque de s'y perdre, école du don au temps de son propre temps, mais également école de l'éducation et de la transmission d'une façon d'être à nos jeunes pousses, ne demandant qu'à absorber des savoirs et des dogmes pour mieux les contester.

Aimer la recherche, c'est aussi aimer la recherche publique - académique écriraient nos collègues étrangers - et ce qui la caractérise, la reconnaissance de son caractère libre, sans utilité fonctionnelle immédiate, dont le terme des innovations sous-jacentes à échoir est parfaitement inconnu, proche ou lointain, liberté et désintéressement qui nous donnent droit aux erreurs et aux tâtonnements, voire aux échecs, source de frustration et d'anxiété dans les laboratoires placés sous le joug implacable des facteurs d'impact, habiles fruits des batailles entre majors de l'édition scientifique, sous le joug de classements mondiaux paramétrés par l'intelligence non artificielle de quelques demiurges intéressés.

Aimer la recherche, c'est aussi reconnaître qu'elle a des devoirs et des responsabilités: devoir de transfert de ces nouvelles connaissances et de nouveaux concepts vers le reste de la société, devoir d'éducation des générations les plus jeunes, devoir d'explication et de contribution au bien-être social de notre monde, et, en ce qui concerne le champ de la recherche biomédicale, à l'amélioration de la santé. Cette dernière doit faire sienne ce devoir de regard clinique, d'intégration de ce va-et-vient essentiel entre médecine et sciences, entre recherche clinique et recherche fondamentale, cet aller-retour géniteur de savoirs fondamentaux et de diagnostics et de thérapies nouvelles et efficaces. Mais la recherche biomédicale, comme d'autres, est plus que jamais l'objet de fractures et d'enjeux: moments de fractures éthiques et bioéthiques, qui déchirent le voile de mondes nouveaux, où le vertige de la puissance créatrice démultipliée par de nouveaux outils et de nouvelles approches - édition du génome ou entrisme dans nos cerveaux, données en masse et ordinateurs quantiques - pourrait nous amener à une rupture du contrat social, lourde de conséquences, avec son rejet du monde scientifique et de sa parole, son rejet de la recherche et, paradoxalement, une irruption massive d'une incrédulité et irrationalité sauvage, conduisant au refus de tout intellectualisme et au mépris de ceux qui en sont les porteurs. Femme ancrée dans la terrible réalité des faits, Simone Veil, lors de la célébration du $X^{e}$ anniversaire de l'Inserm, déclarait «... la recherche biomédicale est, dans une nation, le fondement indispensable d'une médecine de qualité », une déclaration que l'on peut aisément extrapoler à l'ensemble de la recherche, fondement indispensable d'une société éclairée, au sens des Lumières. 
Enjeux économiques, car les découvertes issues de la recherche biomédicale créent de facto de formidables opportunités de développement économique au travers des innovations qu'elles suscitent. $\varepsilon$ n ce sens, la recherche, internationaliste et collaborative, est aussi compétition, compétition entre ego, entre laboratoires, entre industries, entre pays. Elle est donc un enjeu, qui se traduit par la capacité de telle ou telle institution à construire des ponts entre recherche et innovation valorisée, transfert de technologies où la protection intellectuelle des inventions doit aller de pair avec une prospection extensive de partenaires industriels, voire leur création, ainsi que par la capacité de tel ou tel pays à favoriser structurellement et financièrement l'émergence d'un tissu d'entreprises créatrices d'emplois. La recherche ne vit donc pas dans un monde éthéré. La recherche publique est un métier, avec ses règles, ses codes, ses fonctionnements et ses financements. Elle rassemble des savoirs, des savoir-être, et des savoir-faire, elle nécessite une continuité et un continuum. Des permutations internes, mais pas d'expulsions. Scandée par des ruptures cognitives, elle nécessite paradoxalement une stabilité humaine dans la durée. Elle nécessite l'irruption de la jeunesse et de ses paris, mais aussi la construction d'édifices intellectuels et organisationnels stables, constructions qui requièrent du temps et de l'argent. Guillaume Budé, à l'origine de la création en 1530 du Collège royal, futur Collège de France, et Francis Bacon, qui a posé an Angleterre les bases d'une recherche scientifique institutionnalisée, l'avaient compris en leur temps. La recherche n'est pas une abstraction, ce sont des laboratoires, des hommes et des femmes, qui constituent tout un tissu social diffusant de la connaissance et de l'innovation au travers de l'ensemble d'un pays, qu'il s'appelle France ou États-Unis. Grave erreur que celle consistant à penser que seuls quelques points «d'élite » font la recherche d'un pays. Ils ne sont que le sommet brillant et excellent d'une pyramide, ils ne sont que l'émanation ultime de ce tissu. Déchirer le tissu, et la pyramide s'effondrera, ainsi que son sommet.

De tout cela, médecine/sciences, son équipe et son comité éditorial, continueront à vous informer, à vous entretenir et à en alimenter les débats, en donnant la parole à nos collègues francophones de tous les pays.

Avec nos meilleurs vœux de succès pour cette nouvelle année! Que continue de vivre en 2020 l'amour de la recherche dans médecine/sciences! $\diamond$

médecine/sciences listening to research

\section{LIENS D'INTÉRÊT}

L'auteur déclare n'avoir aucun lien d'intérêt concernant les données publiées dans cet article.

\section{Jean-Luc Teillaud \\ Rédacteur en chef de médecine/sciences Directeur de recherche émérite à l'Inserm Équipe «Microenvironnement immunitaire et immunothérapie » Inserm UMRS 1135 \\ Sorbonne université 91, boulevard de l'Hôpital 75013 Paris, France jean-luc.teillaud@inserm.fr} Centre d'immunologie et des maladies infectieuses (CIMI)

TIRÉS À PART

J.L. Teillaud

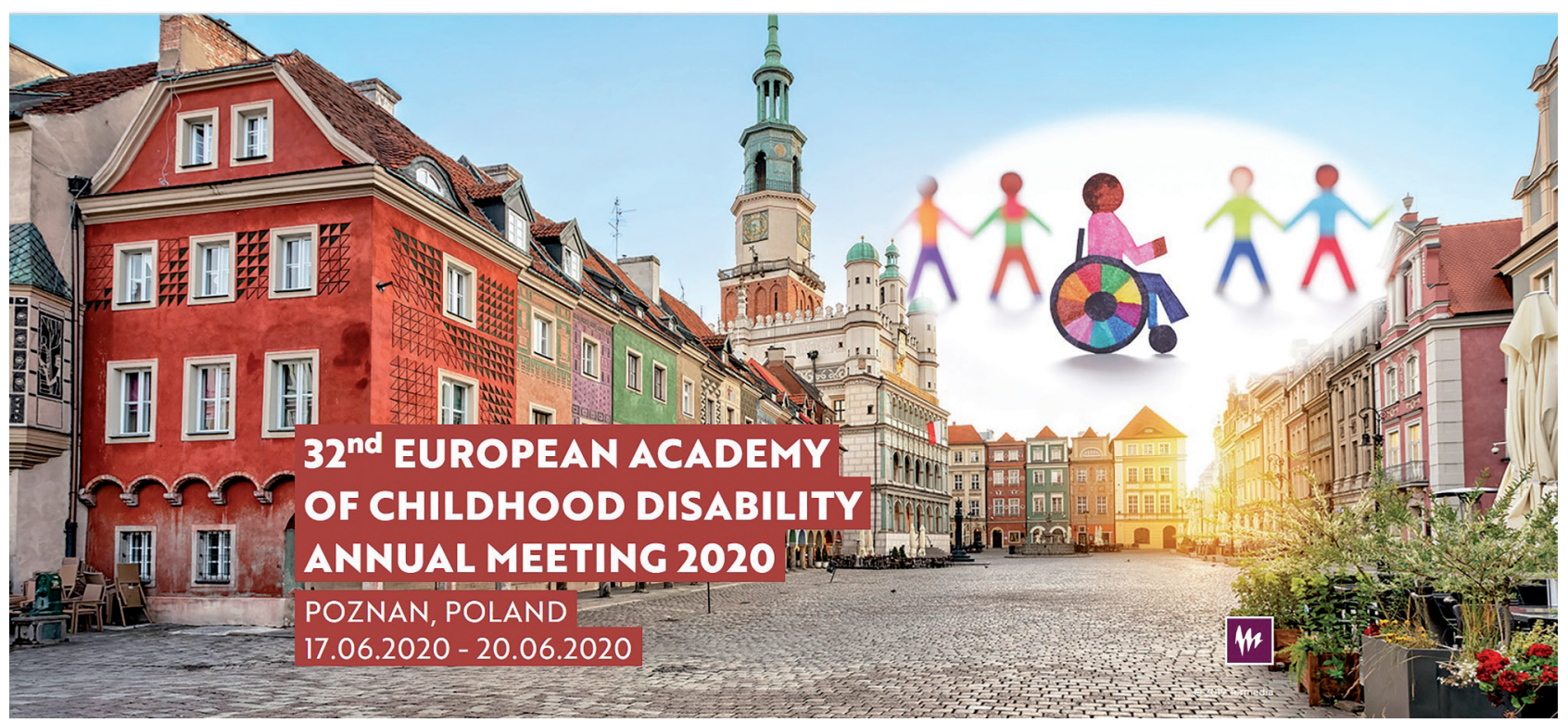

